\title{
HR-TEM and EELS Studies of Soluble Aminopolymer-Functionalized Single-Walled Carbon Nanotubes
}

\author{
Y. Lin,* D. E. Hill,* J. Bentley,** L. F. Allard** and Y.-P. Sun*
}

*Department of Chemistry and Center for Advanced Engineering Fibers and Films, Howard L. Hunter Chemistry Laboratory, Clemson University, Clemson, SC 29634-0973, **Metals and Ceramics Division, Oak Ridge National Laboratory, Oak Ridge, TN 37831-6064

Functionalization and solubilization of carbon nanotubes has attracted significant interest recently since it allows processing of the nanotubes in a controllable fashion and thus provides opportunities for many applications [1,2]. Both solution-phase and solid-phase techniques were applied to the characterization of these functionalized carbon nanotubes. Among them, TEM is one of the most widely used tools because it provides not only global information on nanotube purity and dispersity, but also near-atomic-level information on nanotube surfaces and interiors.

We have used an aminopolymer, poly(propionylethylenimine-co-ethylenimine) (PPEI-EI, $M_{\mathrm{W}}$ 200,000), to functionalize single-walled carbon nanotubes (SWNTs) by a typical defect-targeted amidation reaction [2]. The functionalized SWNTs are soluble in chloroform and water, and the solution appears black at high concentration but is still homogeneous. Typical TEM specimens were prepared by applying a few drops of PPEI-EI-functionalized SWNT aqueous solution to holey carbon-coated copper grids, and were examined in a Hitachi HF-2000 FE-TEM, operated at $200 \mathrm{kV}$. As shown in Fig. 1, the functionalized SWNTs are well-dispersed in the solution with average length of $\sim 500 \mathrm{~nm}$. At higher magnification, individual and bundles of nanotubes covered by amorphous materials were found (Fig. 2). Since polymer molecules are responsible for the solubilization of SWNTs, it seems reasonable to suggest that the amorphous materials on the nanotube surface are polymers. However, it is known that the as-produced SWNTs usually contain substantial amounts of amorphous carbon material. A purification procedure prior to the functionalization reaction efficiently eliminates these amorphous carbons but never completely. Since the polymer we use contains significant nitrogen (N/C 0.22), N presence in the amorphous material found on the SWNT surface would provide proof of the presence of the polymer. Because energy dispersive spectroscopy was not successful in detecting $\mathrm{N}$, electron energy loss spectroscopy techniques (EELS) in a FEIPhilips CM-200F TEM were employed.

The K-edges of $\mathrm{C}$ and $\mathrm{N}$ in the EELS spectra are $284 \mathrm{eV}$ and $401 \mathrm{eV}$, respectively. This allows good resolution between $\mathrm{C}$ and $\mathrm{N}$ signals regardless of the broad feature of C-K EELS core loss. Figure 3 shows (red trace) a typical EELS spectrum for a PPEI-EI-functionalized SWNT bundle. The N/C ratio is estimated to be 0.08; spectra from other areas gave values in the range of 0.07-0.10. Therefore, the amorphous material on the SWNTs is associated primarily with the aminopolymer used for functionalization. Some of the material was dispersed onto a holey $\mathrm{LaCrO}_{3}$-coated film on a stainless steel grid, and heated ex-situ in air at $370^{\circ} \mathrm{C}$ for $\sim 15 \mathrm{~min}$ to remove the polymer selectively. After this treatment, the nanotube surfaces were found to be almost free of amorphous material (Fig. 4), while EELS confirmed that N/C ratio dropped to $\sim 0.02$ (blue trace, Fig. 3). 
In conclusion, we demonstrate that a combination of HR-TEM and EELS can serve as a powerful tool to provide direct information on the structure of soluble functionalized SWNTs at the individual nanotube level, especially those with functionalities containing heteroatoms. [3]

\section{References}

[1] Chen, J.; Hamon, M. A.; Hu, H.; Chen, Y.; Rao, A. M.; Eklund, P. C.; Haddon, R. C. Science 1998, $282,95$.

[2] Sun, Y.-P.; Fu, K.; Lin, Y.; Huang, W. Acc. Chem. Res. 2002, 35, 1096.

[3] Research sponsored by Asst. Secretary for Energy Efficiency and Renewable Energy, Off. of FreedomCAR and Vehicle Technologies, as part of the HTML User Program, ORNL, managed by UT-Battelle LLC for the US DOE. Y.-P.S. acknowledges NSF, NASA, the South Carolina Space Grant Consortium, and the Center for Advanced Engineering Fibers and Films (NSF-ERC at Clemson University) for financial support.

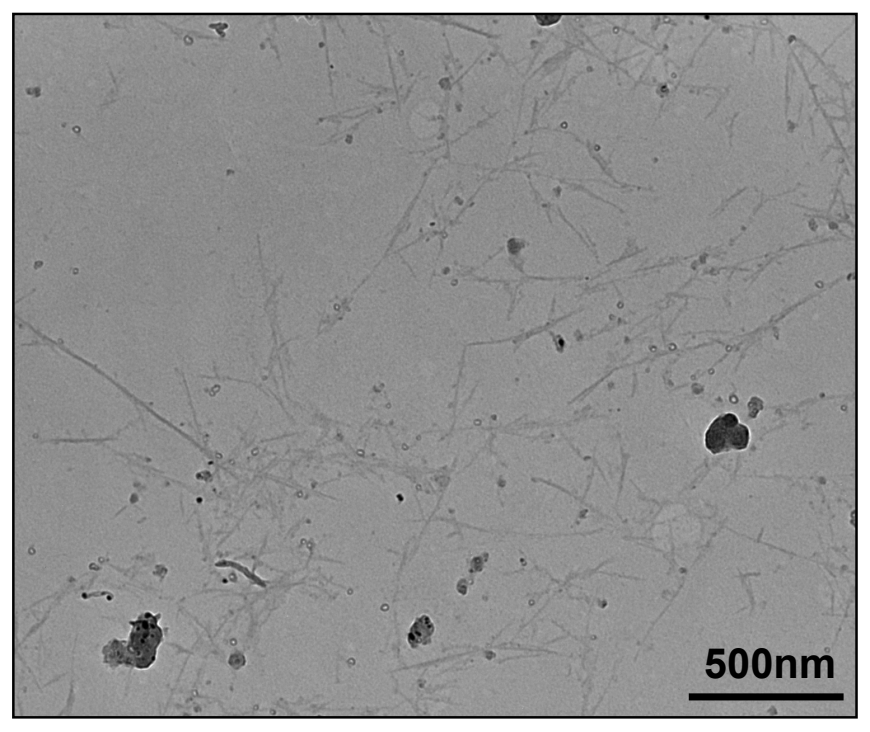

FIG. 1. A low magnification TEM image of PPEI-EI-functionalized SWNTs.

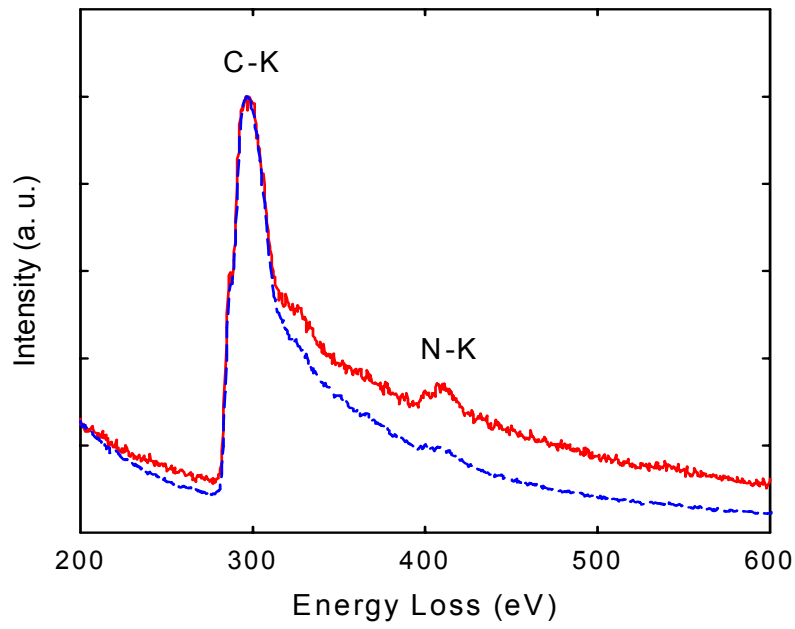

FIG. 3. Typical EELS spectra of a PPEI-EI-functionalized SWNT bundle before (- - and after (-----) ex-situ thermal treatment.
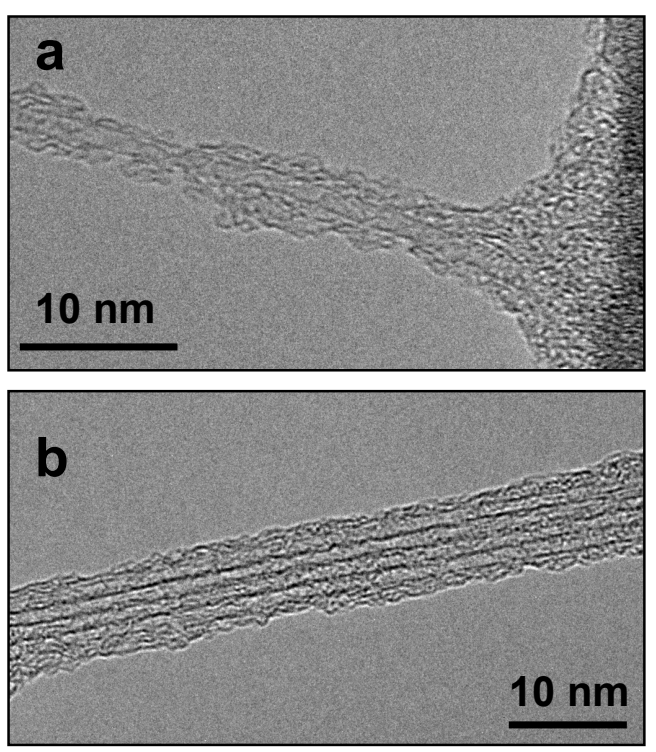

FIG. 2. HR-TEM images of (a) an individual and (b) a bundle of PPEI-EIfunctionalized SWNTs.

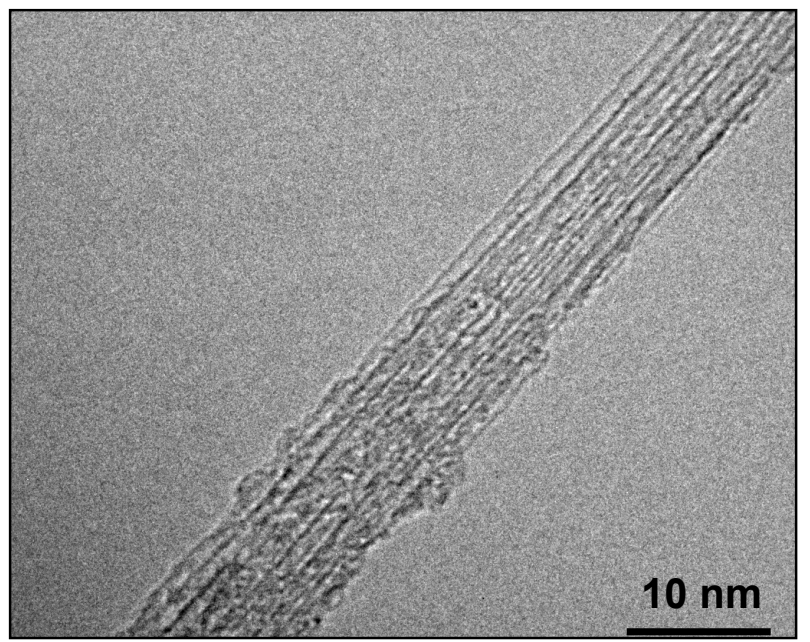

FIG. 4. HR-TEM image of a bundle of PPEI-EIfunctionalized SWNTs after ex situ thermal treatment. 